\title{
Highlights of the expansion of SN1993J
}

\author{
Jon Marcaide ${ }^{* a}$ and Iván Martí-Vidal ${ }^{b}$ \\ ${ }^{a}$ Universidad de Valencia \\ E-mail: marcaide@uv.es \\ ${ }^{b}$ Onsala Space Observatory \\ E-mail: ivan.marti-vidalechalmers.se
}

SN 1993J in M 81, a powerful radio supernova high in the northern sky, has been carefully monitored with VLBI throughout its lifetime. Its extremely circular shell-like radio structure has expanded over 15 years in a rather self-similar way in reasonable agreement with Chevalier's model. An extension of Chevalier's model simultaneously accounts for all the light-curve and VLBI results. Beyond the first year a single deceleration parameter, $m_{\text {true }}=0.87 \pm 0.02$, characterizes the shock expansion. However, at short wavelengths and beyond 5 years, an enhanced deceleration, $m_{\text {shortwavelengths }}=0.79 \pm 0.01$, is measured. This enhancement is interpreted as due to a combination of effects ranging from varying free-free opacity in the supernova ejecta at the short wavelengths to the radial shape of the intensity of the magnetic fields in the emitting region. The final radio-loud stage is characterized by an abrupt decrease of radio emission. This large flux-density decay rate can be explained as due to the supernova shock surpassing the outer boundary of the circumstellar medium generated by the supernova progenitor. Presently, the supernova expansion beyond the circumstellar/interstellar boundary is rather radio silent, although there are indications that the supernova remnant could be studied by LOFAR and eMERLIN.

Resolving the Sky - Radio Interferometry: Past, Present and Future -RTS2012

April 17-20, 2012

Manchester, $U K$

*Speaker. 
Foreword by Jon Marcaide: I first met Richard Schilizzi in 1979 and we started soon a lasting friendship. Over all these years we collaborated in various ways, also in scientific projects. One of those projects was the "VLBI study of the expansion of SN1993J", a major effort which spanned from 1993 through 2011. We published about 10 refereed papers in this project and Richard was coauthor of 4 of those. This is why I have chosen this contribution to honor his "retirement". Many other people 1 collaborated at different stages in this effort. In particular, more than half of my grad students were involved. All of them made strong contributions. However, I would like to make a special mention to the key contributions of Alberdi in the early phase and Martí-Vidal in the final phase. Because so much of the final analysis and conclusions of our decade-long effort relies on the work of Marti-Vidal, I have thought appropriate to write this contribution with him.

\section{Introduction}

Supernova SN 1993J exploded on 1993 March 28. By mid April, soon after the first radio detection, it was clear that SN 1993J was a powerful type II radio supernova. It was a core-collapse supernova with a red giant progenitor in the nearby galaxy, M81, at a high declination in the northern hemisphere sky. It was the kind of radio supernova one had been waiting for to make a thorough VLBI study. It was the ideal radio supernova to test Chevalier's model [2] [3] in gory detail. In Chevalier's model, the radio emission is due to non-thermal synchrotron generated by relativistic electrons as they interact with the magnetic field in the shocked circumstellar medium (CSM). The electrons are accelerated to relativistic speeds and the magnetic field is intensified by the expanding shock of the supernova. In Chevalier's model, the radio-emitting region has the shape of a spherical shell (hence the alternative name of mini-shell model) and the expansion is self-similar (i.e., the structure at a given epoch is just a scaled version of the structure at any other epoch). The radius of the spherical shell, $R$, is proportional to $t^{m}$, a power law of the supernova age $t$, where $m$ is the so-called deceleration parameter. It can be shown that $m=(n-3) /(n-s)$ (where $n$ and $s$ are the exponents of the power laws that describe the radial density profiles of the ejecta and the CSM, respectively).

\section{Early results}

The most basic assumption of the mini-shell model was confirmed with our discovery of the shell-like radio structure (see Fig. 1 left) from VLBI observations carried out at $3.6 \mathrm{~cm}$ on day 239 after explosion [5]. The self-similar expansion was also confirmed with data from the first 500 days [6] (see Fig. 1 right). The first determination of the value of the deceleration parameter had to await to the availability of 3 years of VLBI data [7]. The value determined, $m=0.86 \pm 0.02$, allowed us to estimate the power index of the ejecta density profile, $n$, assuming a standard CSM profile (i.e., $s=2$ ). However, at that time, there was some confusion with the interpretation of the early $\mathrm{X}$-ray results, and it was debated whether the CSM profile corresponded to a value of $s=2$ (i.e., the expected value that would result from a constant stellar wind of the progenitor) or $s=1.6-1.7$

${ }^{1}$ A. Alberdi, L. Bååth, R. Davies, G. de Bruyn, P. Diamond, P. Elósegui, J. Guirado, D. Jones, T. Krichbaum, L. Lara, F. Mantovani, I. Martí-Vidal, E. Pérez, M. Pérez-Torres, R. Preston, A. Rius, A. Rogers, E. Ros, B. Schmidt, I. Shapiro, R. Schilizzi, R. Sramek, C. Stockdale, S. van Dyk, K. Weiler, A. Whitney, A. Witzel, C. Trigilio, A. Zensus 
(i.e., due to a stellar wind with mass-loss rate decreasing with time). Time settled the question to the value of $s=2$ (e.g., [4]), and hence the value of $n$ was reliably determined.

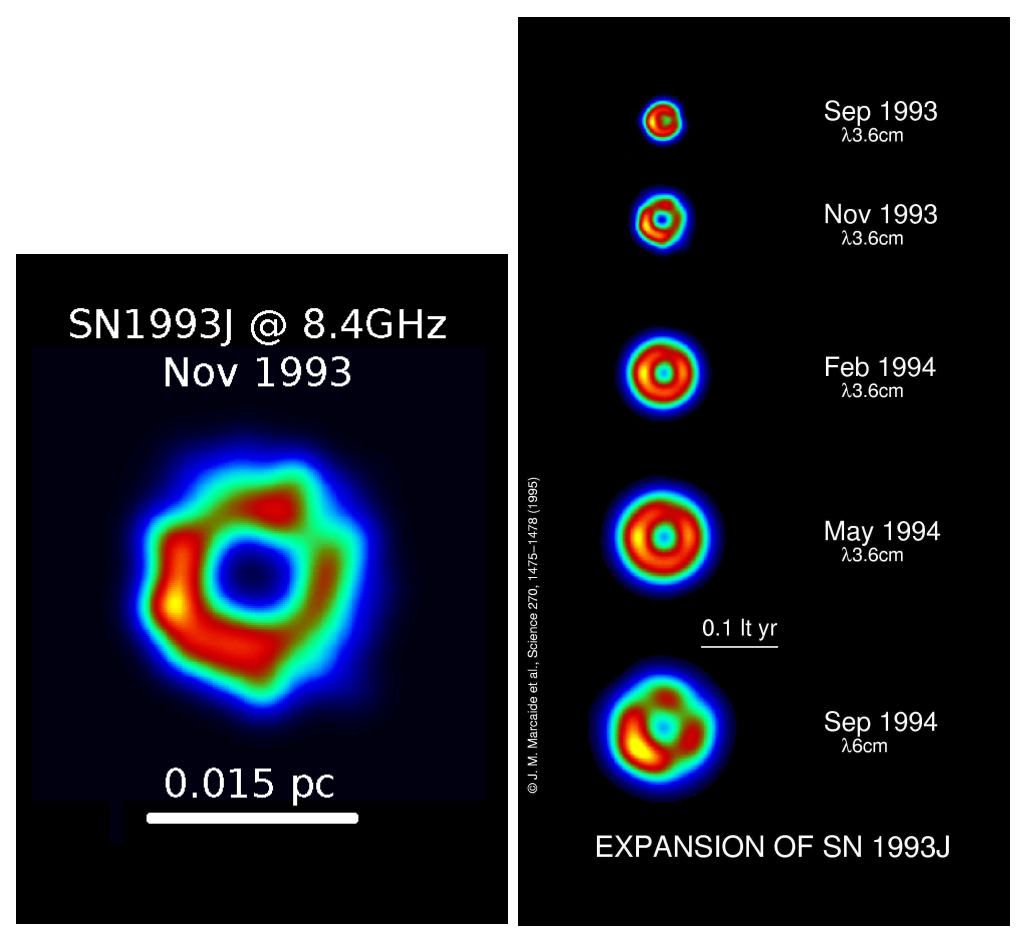

Figure 1: Left, figure of the discovery of the shell-like radio structure of SN 1993J. Right, self-similar expansion of SN 1993J.

\section{Long journey}

With the main features of Chevalier's model confirmed it remained to study the expansion with high precision in order to test possible departures from that model. The expansion was taking place rather self-similarly, but regions of slightly enhanced emission in the shell structure were apparent and changing. Also, the width of the shell was an important parameter to be determined. However, determining the width of the shell was not easy task and we dedicated much effort to it. To make things more difficult, we also found out that the emission from far side of the shell was absorbed by the ionized ejecta and hence an overall optically-thin model could not be used to estimate the shell width. We made an initial determination of both the shell width and the ejecta opacity using novel methods [8]. At about the same time, a competing team [1], which had been making VLBI observations as often as us, but using a somewhat different strategy, came to similar conclusions to ours, although they analyzed their data with different tools and in Fourier space. They estimated shell widths much narrower than we $\operatorname{did}(\sim 0.2$ times the outer shell radius, compared to our findings of $\sim 0.3$ ).

Since the circularity of the shell was remarkably high (eventually we estimated the departures from circularity to be under 2 percent), we started to develop new analysis tools using the circular symmetry to our advantage. These new tools would allow us to measure with high precision the 
supernova size in the sky plane. As a consequence of our results with the new method, at some point around year 2006 we started to have slight discrepancies in the interpretation of results with our colleagues. With the new tools we had developed to measure the circular shell size, we could clearly see what in a Fourier analysis appeared only as a hint, namely, that at the wavelength of $18 \mathrm{~cm}$ the source appeared larger than at $6 \mathrm{~cm}$. Actually, the story goes the other way around: both teams could measure a progressive change of expansion rate at $6 \mathrm{~cm}$ beyond approximately day 1500 after explosion. For us, the enhanced deceleration, while clear at $6 \mathrm{~cm}$, was not taking place at the longer wavelength of $18 \mathrm{~cm}$. However, if the data at 6 and $18 \mathrm{~cm}$ were analyzed jointly, the data at late epochs (with relatively more observations at $18 \mathrm{~cm}$ ) could be interpreted as corresponding to a re-acceleration in the expansion [1]. Our colleagues supported this interpretation as being compatible with that expected from hydrodynamic simulations using a very singular explosion model. For us, there was no re-acceleration in the expansion at all, and the different evolution in the expansion at 6 and $18 \mathrm{~cm}$, of which we had firm evidence (see next section), was a manifestation of the characteristics of the emitting and absorbing regions (an evolving and frequency-dependent free-free ejecta opacity for the latter and a non-uniform shape of the magnetic-field intensity in the radio-emitting region for the former).

In [9] we presented the analysis of all our VLBI data, which consisted of over thirty VLBI runs at 4 frequencies. We estimated the supernova sizes using the Common Point Method (CPM, [10]) which, as said, we developed to measure with high precision in the sky plane taking advantage of the extreme circular symmetry of the source. The expansion is shown in Figure 2. We found that the expansion was characterized for the first 5 years (we had no data prior to day 181) by a deceleration parameter ( $m=0.845 \pm 0.005)$ which happened to be the same as the expansion observed at $18 \mathrm{~cm}$ for the remaining years (until the radio source started to rapidly fade out). However, at shorter wavelengths the deceleration was enhanced $(m=0.788 \pm 0.015)$. We noticed also, in our VLBI flux densities, that appearing about 5 years after explosion there was an excess of emission at the shorter wavelengths. This excess of flux density at high frequencies could also be observed in the multi-frequency flux-density monitoring of Weiler et al. [13]. In the next section, we explain how both effects (i.e., a slight over-emission at higher frequencies coupled to an enhanced deceleration in the expansion observed at the same frequencies) can be explained using one single model.

\section{Global analysis and simulations}

Having two sets of data taken by different teams with different observing strategies, analyzed differently and interpreted differently, did not look to us as the ideal situation, even though the main results (i.e., a self-similar expansion following a power-law of time) were similar. Actually, we had analyzed our data both using the CPM in the sky plane and conventional fitting in Fourier space (perhaps, the term conventional is not the appropriate term here, given that the model to be fitted presented parameters, like shell width or degree of optical thickness in the ejecta, that were not well known a priori). Hence, we decided to undertake the enormous effort of reanalyzing our data and the data of our colleagues (retrieved by us from the VLBA archives) from scratch in a very uniform manner with a strategy designed to minimize biases and be able to compare results obtained with different methods. In this reanalysis, we used measurement methods in the sky plane 


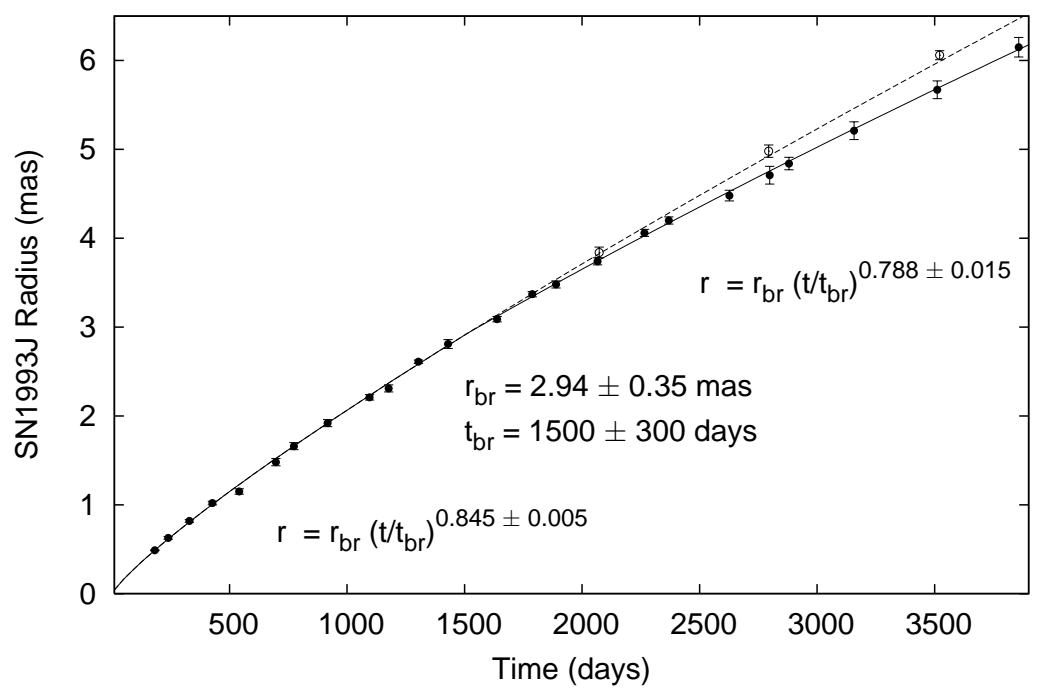

Figure 2: Expansion of SN 1993J over a decade, based solely on our data. The deceleration rate remains unchanged at $18 \mathrm{~cm}$ (empty circles) while at $6 \mathrm{~cm}$ (filled circles) the deceleration is enhanced, thus providing valuable information about the physical parameters in the emitting region.

and in Fourier space. Most importantly, we also developed a code, $R A M S E S^{2}$, to generate realistic simulations of the radio emission from the supernova [10] [12]. In this code, an extended numerical Chevalier model, we included effects that were not considered in the original formulation of the model and which could affect the results of both expansion curve and flux-density evolution at the high-precision level achievable in the observations. These effects included radiative and inverseCompton losses, evolving free-free absorption in the ejecta, possible non-uniform amplification of the magnetic field in the shocked region of the CSM, etc. Given that we also had a complete set of light curves available at wavelengths ranging from $3 \mathrm{~mm}$ to $90 \mathrm{~cm}$ [13], we planned to use the RAMSES code to fit simultaneously the VLBI expansion curves and the radio light-curves at all frequencies.

The analysis of the two sets of VLBI data (over thirty VLBI runs spanning almost 15 years in each set) gave results similar to those previously reported by us [9]. Figure 3 shows the final result using the joint dataset and the CPM. Analyzing the datasets separately, or using model fitting in Fourier space instead of the CPM, gives essentially the same results if we consider the small biases between the methods (see [11] for details). Figure 3 shows the excellent fit of the simulations to both the VLBI data and the radio light curves at all frequencies. We would like to emphasize again that both fits are obtained simultaneoulsy (i.e., using a single model).

Table 1 summarizes the best-fit parameters obtained in the fit of RAMSES to the global VLBI data set (66 epochs of observation) and to all the radio light-curve data. For a more detailed explanation on the meaning of these parameters, see [12].

Two main results of our analysis of the joint dataset are summarized in the following subsections. Other relevant results (e.g., the determination of the shell width size, the structure of the shell inhomogeneities and its evolution, the evidence of CSM clumpiness from the early opacity evolution, or a "flare" resulting from a large over-density in the CSM at 1150 AU from the progenitor)

\footnotetext{
${ }^{2}$ Radiation-Absorption Modelling of the Synchrotron Emission from Supernovae
} 


\begin{tabular}{c|c|c} 
Expansion curve & Particles \& fields & CSM density \& temperature \\
\hline$m_{\text {true }}=0.87 \pm 0.02$ & $\bar{B}_{0}=65.1 \pm 1.6 \mathrm{G}$ & $n_{0}=(6 \pm 0.9) \times 10^{8} \mathrm{~cm}^{-3}$ \\
$m_{\text {shortwavelengths }}=0.79 \pm 0.01$ & $f_{\text {rel }}=(5 \pm 0.5) \times 10^{-5}$ & $T_{l}=(2.0 \pm 0.1) \times 10^{6} \mathrm{~K}$ \\
& $p=-2.55 \pm 0.01$ & $t_{m}=2500 \pm 100$ days \\
\hline
\end{tabular}

Table 1: Summary of RAMSES fitted parameters for SN 1993J. The physical conditions are given for a reference supernova age of 5 days. The deceleration parameters apply for ages beyond 360 days (see [12] for details).
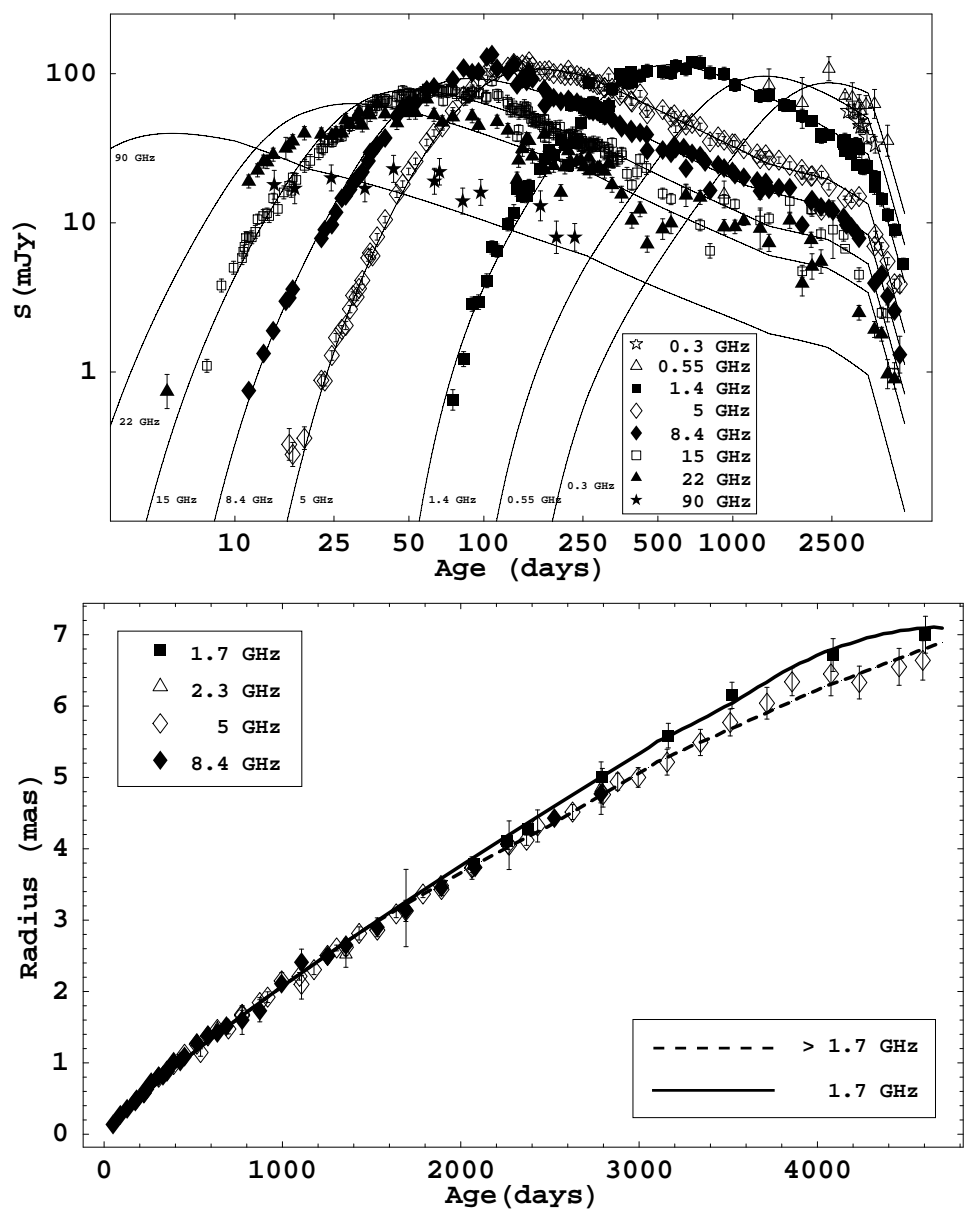

Figure 3: Above: radio light curves of SN 1993J. Below: expansion curves of SN 1993J obtained after a reanalysis of 66 (all the available) VLBI observation epochs of SN 1993J at 18, 13, 6, and $3.6 \mathrm{~cm}(1.6,2.3$, 5.0, and $8.4 \mathrm{GHz}$, respectively) For both simultaneously, best-fit RAMSES model (above: solid line; below: solid line for the $18 \mathrm{~cm}$ data and dashed line for data at shorter wavelengths).

which are too long to be explained in these proceedings, are described in [9], [11], and [12].

\subsection{Changing opacity in the ejecta}

In Figure 4, we show the evolution in the spectral index at 1.6 and $5.0 \mathrm{GHz}$ [13], compared to the RAMSES model (continuous line) and to the analytical model reported in the quoted paper 
(dashed line). A flattening in the spectrum at late epochs (i.e., a decrease in the absolute value of the spectral index) can be appreciated in the figure, which also shows how the RAMSES model fits remarkably well the evolution in the spectral index at all epochs. The flattening in the spectrum at late epochs is modelled by RAMSES as due to a changing opacity in the ejecta (i.e., the same effect that allows RAMSES to fit the different VLBI expansion curves at different wavelengths). This flattening cannot be adequately modeled with the model presented in [13].

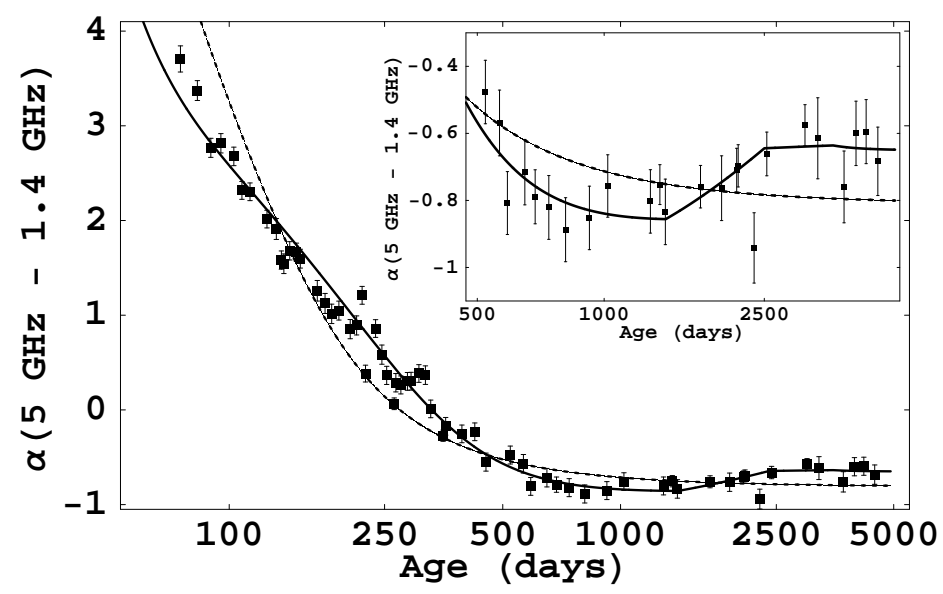

Figure 4: Evolution in the spectral index of SN 1993J, between 1.6 and 5.0 GHz. The dashed line is the best-fit model of Weiler et al. (2007) and the continuous line is the RAMSES model fit. The inset is a blow-up of the figure for the latest epochs.

\subsection{Radio-death: evolution into a supernova remnant}

In [13], Weiler et al. report an enhanced flux-density decay rate in their light curves at all frequencies in their late data (from day $\sim 3100$ after explosion onwards). These authors explain such a decay as due to an exponential fall in the CSM radial density profile. Such a rapid fall in the CSM density could be due to the boundary of the CSM which is related to the onset of the strong wind of the progenitor. However, RAMSES is able to reproduce this enhanced decay as just due to the synchrotron ageing of the electrons, as long as we assume that the expanding shock passed through a sharp boundary from the CSM into the interstellar medium (ISM). In other words, radiative losses, alone, allow us to explain the observed enhancement in the flux-density decay at late epochs, without the need of a soft exponential-like decay of the CSM density, but just a well-defined boundary with the ISM. However, "softer" transitions from the CSM to the ISM would also be compatible with the data, as long as other parameters in the simulation (e.g., escaping of the electrons from the emitting region) are introduced. We notice though that the CSMISM transition boundary (whose real structure depends on the particulars of the onset of the stellar wind in the progenitor star) must be, in any case, sharper than the exponential-like decay claimed in [13].

\section{Conclusions}

We have highlighted the main results obtained from the VLBI monitoring of supernova SN 1993J: 
from the discovery of the shell-like radio structure and the study of its self-similar expansion to the discovery of a wavelength-dependent expansion (mainly explained by a changing ejecta opacity) and its relationship to unmodelled effects in the radio light curves (a flattening in the spectrum at late epochs). Observations obtained by another research group, who reached different conclusions on the details of the expansion curve, are compatible with our results after a homogeneous reanalysis, even after using different approaches to estimate the supernova shell size. We explain the difference between our and their conclusions as due to subtle biases related to the data analysis strategy.

Even though some years have elapsed since the last reported radio observations of SN 1993J, and the exponential-like flux-density decay may have continued since then, SN 1993J could still be detected with new available instrumentation (e.g., eMERLIN and LOFAR). The minimum expected flux density at $\mathrm{GHz}$ frequencies is a fraction of a mJy (detectable with MERLIN or the extended VLA). According to our simulation software, SN 1993J should be currently peaking at about $250-300 \mathrm{MHz}$ at the level of 1.5-2.0 mJy and it could perhaps be a good target for LOFAR. New monitoring of the flux-density decay with these arrays could bring valuable information on the dynamics of the shock in the circumstellar/interstellar transition region and on the interstellar region in $\mathrm{M} 81$.

\section{Multimedia}

Some graphic material can be found at our website ${ }^{3}$. Three movies of the supernova expansion at different stages and two composite figures of the expanding shell have been produced.

\section{References}

[1] Bartel, N., Bietenholz, M. F., Rupen, M. P., et al. 2002, ApJ, 581, 404

[2] Chevalier, R. A. 1982a, ApJ, 258, 790

[3] Chevalier, R. A. 1982b, ApJ, 259, 302

[4] Nymark, T. K., Chandra, P, \& Fransson, C. 2009, A\&A, 494, 179

[5] Marcaide, J. M., Alberdi, A., Ros, E., et al. 1995a, Nature, 373, 44

[6] Marcaide, J. M., Alberdi, A., Ros, E., et al. 1995, Science, 270, 1475

[7] Marcaide, J. M., Alberdi, A., Ros, E., et al. 1997, ApJ, 486, 31

[8] Marcaide, J. M., Martí-Vidal, I., Ros, E., et al. 2005, in Cosmic Explosions, IAU Colloq. 192, ed. J. M. Marcaide \& K. W. Weiler (Berlin:Springer) 29

[9] Marcaide, J. M., Martí-Vidal, I., Alberdi, A., et al. 2009, A\&A, 505, 927

[10] Martí-Vidal, I. 2008, PhD. thesis (University of Valencia, Spain)

[11] Martí-Vidal, I., Marcaide, J. M., Alberdi, A., et al. 2011, A\&A, 526, A142

[12] Martí-Vidal, I., Marcaide, J. M., Alberdi, A., et al. 2011, A\&A, 526, A143

[13] Weiler, K. W., Williams, C. L., Panagia, N., et al. 2007, ApJ, 671, 1959

\footnotetext{
${ }^{3}$ http://www.uv.es/radioast/main/multimedia
} 